\title{
Coma e outros estados de consciência
}

\section{Coma and other consciousness disorders}

\begin{abstract}
Almir Ferreira de Andrade', Rodrigo do Carmo Carvalho', Robson Luis Oliveira de Amorim ${ }^{3}$, Wellingson Silva Paiva ${ }^{4}$, Eberval Gadelha Figueiredo ${ }^{5}$, Manoel Jacobsen Teixeira ${ }^{6}$
\end{abstract}

\begin{abstract}
Andrade AF de, Carvalho RC, Amorim RLO de, Paiva, WS, Figueiredo EG, Teixeira MJ. Coma e outros estados de consciência. Rev Med (São Paulo). 2007 jul.-set.;86(3):123-31.

RESUMO: As alterações do nível de consciência podem variar entre dois extremos, desde uma desorientação têmporo-espacial até um estado de coma profundo. Na prática clínica é extremamente comum o médico se deparar frente a um paciente com alteração do nível de consciência. Em hospitais gerais, estudos indicam o alcoolismo, o traumatismo cranioencefálico e as doenças cardiovasculares como as principais causas de coma (82\%). O médico deve saber reconhecer as principais causas de alteração da consciência, seus diagnósticos diferenciais, identificar o paciente grave e tratar as possíveis alterações emergenciais que possam levar a um dano neurológico secundário. Nesta revisão destacamos os principais pontos no exame neurológico para determinar as diferentes alterações do nível de consciência.
\end{abstract}

DESCRITORES: Coma/diagnóstico. Coma/fisiopatologia. Traumatismos encefálicos/etiologia. Consciência/classificação. Diagnóstico diferencial.

\footnotetext{
1. Prof. Livre docente e Coordenador da Equipe de Neurocirurgia de Emergência, Hospital das Clínicas da Faculdade de Medicina da Universidade de São Paulo.

2. Departamento de Neurologia, Hospital das Clínicas da Faculdade de Medicina da Universidade de São Paulo.

3. Divisão de Neurocirurgia, Hospital das Clínicas da Faculdade de Medicina da Universidade de São Paulo.

- Divisão de Neurocirurgia, Hospital das Clínicas da Faculdade de Medicina da Universidade de São Paulo.

. Divisão de Neurocirurgia, Hospital das Clínicas da Faculdade de Medicina da Universidade de São Paulo.

6. Professor Titular da Disciplina de Neurocirurgia, Hospital das Clínicas da Faculdade de Medicina da Universidade de São Paulo.

Endereço para correspondência: Wellingson Silva Paiva. Rua Dr. Ovídio Pires de Campos, 171. Ap. 511. CEP: 05403010. São Paulo, SP, Brasil. e-mail: wellingsonpaiva@yahoo.com.br
} 


\section{INTRODUÇÃO}

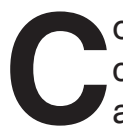
onsciência é definida como a capacidade do indivíduo de reconhecer a si mesmo e aos estímulos do ambiente $^{1,3}$. As alterações da consciência podem se dar no estado de alerta ou nível de consciência ou no conteúdo da consciência, que englobariam as funções mentais e cognitivas do indivíduo. Neste capítulo daremos mais ênfase às alterações relacionadas ao nível de consciência.

As alterações do nível de consciência podem variar entre dois extremos, desde uma desorientação têmporo-espacial até um estado de coma profundo. Coma seria definido como o estado de inconsciência de si mesmo e do ambiente, mesmo após estímulos de diversas modalidades e intensidades, em que 0 paciente permanece de olhos fechados ${ }^{1,3}$. Estados intermediários de alteração da consciência podem anteceder a instalação do quadro e precisam ser reconhecidos. A sonolência ou letargia é considerada um estado de diminuição do nível de consciência em que o paciente consegue ser acordado com estímulos brandos ${ }^{3}$. O estupor é considerado um estado de sonolência mais profunda em que o indivíduo precisa receber estímulos vigorosos e repetidos para despertar ${ }^{3}$.

Entre os estados que levam a alteração do conteúdo da consciência encontra-se o delirium. Caracteriza-se por desorientação, déficit de atenção, sensação de medo, irritabilidade e alterações da percepção de estímulos sensoriais, como as alucinações visuais. As alterações são mais evidentes no conteúdo da consciência, embora os pacientes podem inverter o seu ciclo sono-vigília e alternar períodos de alerta e agitação com períodos de sonolência ${ }^{3}$. A demência seria caracterizada como um quadro de perda permanente e progressiva, em geral, evoluindo em meses a anos, das funções cognitivas, sem alteração do estado de alerta ou nível de consciência ${ }^{1}$.

Alguns estados comportamentais podem ser algumas vezes confundidos com o coma. O estado vegetativo persistente ou síndrome acognitiva é descrito como um estado que pode emergir em pacientes que sofreram lesões graves ao sistema nervoso central, muitas vezes ficando em coma por algum período, em que há um retorno do estado de alerta, mas o paciente permanece com uma incapacidade de reagir ou interagir com estímulos ambientais. Há retorno do padrão de sono-vigília e manutenção das funções vegetativas, mas com quase completa ausência de funções cognitivas. Outros termos têm sido utilizados para exprimir comportamentos semeIhantes como coma vigil, síndrome apálica, coma alfa, morte neocotical ou cerebral ${ }^{1,3}$. Alguns autores têm utilizado termos como estado minimamente consciente, para os pacientes que recuperam algumas funções cognitivas, como a habilidade de seguir comandos simples, presença de gestos ou respostas tipo sim ou não ou verbalização ininteligível ${ }^{3}$.

A abulia é um comportamento em que há uma grave apatia com diminuição ou ausência de comportamento emocional ou mental, em que o paciente nem fala ou se movimenta espontaneamente, embora esteja alerta e reconheça estímulos do ambiente. Geralmente ocorre em pacientes com lesões frontais bilaterais $^{3}$. A catatonia é um estado em que o indivíduo pode ficar mudo, e com diminuição acentuada da atividade motora, geralmente associado a quadros psiquiátricos $^{3}$.

A síndrome de Locked-in (estado de deaferentação) descreve um quadro em que ocorre lesão de fibras descendentes supranucleares e piramidais (fibras cortico-nucleares e cortico-espinhais) em que o paciente apresenta incapacidade de movimentação dos membros, afonia, disfagia e limitação da movimentação horizontal dos olhos. Esses pacientes estão acordados e algumas vezes conseguem se comunicar através de piscamentos, evidenciando que não houve alteração da consciência. Geralmente essa síndrome ocorre em pacientes com trombose da artéria basilar e infarto isquêmico da base da ponte e deve ser diferenciada do coma ${ }^{1,3}$. Quadros que podem se assemelhar são polineuropatias agudas, miastenia gravis e uso de bloqueadores neuromusculares ${ }^{1}$.

Morte encefálica ocorre quando o dano encefálico é tão extenso que não há potencial para recuperação estrutural e funcional do encéfalo e o mesmo não pode manter a homeostase interna (funções cardiovasculares, respiratórias, gastrointestinais e controle da temperatura corpórea) ${ }^{2}$. Embora haja métodos artificiais para preservação de órgãos periféricos (medidas de suporte cardiovascular e ventilação mecânica), um corpo em morte encefálica desenvolve falência cardiovascular em alguns dias, raramente em algumas semanas, apesar do mais meticuloso cuidado intensivo. O que separa, pois o estado de coma do diagnóstico de morte encefálica é a irreversibilidade do último, com repercussões sistêmicas sobre a homeostase de órgãos vitais, baseadas em danos permanentes estruturais focais ou difusos no encéfalo ${ }^{2,4,5}$.

\section{FISIOPATOLOGIA}

As estruturas capazes de manter o indivíduo alerta ou desperto, estão localizados na formação reticular e outras estruturas entre a região pontomesencefálica e o diencéfalo (tálamo e hipotálamo), 
chamada de sistema ativador reticular ascendente (SARA) ${ }^{1}$. Lesões nestas estruturas ou que acometam os hemisférios cerebrais de forma difusa ou multifocal podem levar a alterações do nível de consciência ou até mesmo o coma ${ }^{1,3}$.

Podemos dividir as possíveis causas que podem levar a alterações do nível de consciência ou coma como: alterações simétricas, não estruturais; alterações simétricas estruturais; alterações assimétricas, estruturais ${ }^{3}$.

Entre as alterações simétricas e não estruturais (difusas) podemos incluir intoxicações (chumbo, tálio, metanol, monóxido de carbono), uso de drogas (álcool, sedativos, barbitúricos, anfetaminas), distúbios metabólicos (hiponatremia, hipernatremia, hipoxia, hipercapnia, hipoglicemia, hiperglicemia, hipercalcemia, hipocalcemia, hipertermia, hipotermia, uremia, encefalopatia hepática e outros), infecções (meningite, encefalite), quadros psiquiátricos e outros ${ }^{3}$.

As alterações estruturais (focais) podem ser divididas em supratentoriais e infratentoriais. As lesões estruturais e simétricas incluem: supratentorial - oclusão bilateral da carótida interna, oclusão da artéria cerebral anterior bilateral, hemorragia subaracnóidea, hidrocefalia e infratentorial - oclusão da artéria basilar, tumor de tronco, hemorragia pontina. Entre as alterações estruturais assimétricas estão: supratentoriais - tumor unilateral hemisférico com herniação, hemorragia subdural bilateral, sangramento parenquimatoso (tálamo) e outros e infratentoriais - infarto ou hemorragia de tronco encefálico ${ }^{3}$.

No estudo de Plum e Posner ${ }^{3}$, dos 500 pacientes inicialmente diagnosticados como coma de origem desconhecida, 326 apresentavam disfunções difusas e tóxico-metabólicas do encéfalo. Dos remanescentes 101 apresentavam lesões supratentoriais (77 hemorragias e 9 infartos cerebrais), 67 infratentoriais (infartos de tronco) e 8 causas psiquiátricas.

\section{ABORDAGEM DIAGNÓSTICA}

O manuseio inicial do paciente em coma deve enfatizar os cuidados básicos para manter uma via aérea e ventilação adequada, estabilidade hemodinâmica e outras medidas para minimizar os danos ao encéfalo e a outros órgãos vitais. Devemos excluir causas que precisem de abordagem cirúrgica de urgência e as causas médicas que precisem de tratamento imediato.

A identificação da causa do coma deve se iniciar pela coleta de informações com familiares e pessoas que possam ter presenciado a evolução clínica do paciente. É muito importante obter informações com relação à forma de instalação e evolução do quadro, possível uso de drogas ou substâncias tóxicas, presença de febre, história de trauma, doenças prévias e antecedentes psiquiátricos ${ }^{3}$.

O exame clínico geral deve buscar indícios de condições sistêmicas que possam levar a alterações do nível de consciência ${ }^{3}$. Devem-se obter os sinais vitais, pressão arterial, pulso, freqüência respiratória, temperatura, e nunca esquecer de obter uma glicemia capilar, já que a hipoglicemia uma vez identificada deve ser prontamente revertida com a infusão de glicose a $50 \%$ para evitar danos irresversíveis ao encéfalo. O exame do sistema cardiovascular, pulmonar, gastrointestinal, pele e outros sistemas podem fornecer dados importantes quanto à etiologia do coma.

O objetivo do exame neurológico no coma é ajudar na determinação da causa do coma, ter um parâmetro para seguimento evolutivo e para ajudar na determinação do prognóstico do paciente ${ }^{3}$. Entre os dados do exame neurológico que são mais importantes para a localização e prognóstico estão: (1) nível de consciência; (2) padrão respiratório; (3) tamanho e resposta pupilar a luz; (4) motricidade ocular espontânea ou reflexa e (5) resposta motora esquelética.

\section{Nível de consciência}

A avaliação do nível de consciência deve englobar uma descrição do estado de alerta do paciente, em resposta a estímulos verbais e dolorosos. O objetivo é determinar o grau de alteração do nível de consciência, e ter um parâmetro clínico evolutivo e prognóstico. Deve ser feita de forma seriada e seguindo critérios semelhantes entre os examinadores para efeito comparativo.

A escala de coma de Glasgow é uma escala padronizada utilizada para avaliação do nível de consciência em pacientes vítimas de traumatismo craniencefálico ${ }^{3}$. Consiste em uma tabela de escores que pode variar entre 3 (ausência de abertura ocular, da resposta verbal e da movimentação de extremidades, após estímulos dolorosos) e 15 (sem alteração do nível de consciência). Os parâmetros avaliados são a abertura ocular (escore de 1-4), padrão de resposta motora (escore de 1-6) e padrão de resposta verbal (escore de 1-5) ${ }^{6}$. Os estudos têm mostrado que, para que a pontuação seja confiável, os profissionais precisam completar a pontuação com consistência ao avaliar os efeitos do tratamento clínico. O profissional precisa lembrar que a escala é apenas um parâmetro de avaliação; entretanto, até com suas limitações, ela tem dado uma linguagem 
comum para definir o estado da lesão cerebral ${ }^{7,8,9}$.

A escala de coma de Jouvet utiliza como parâmetros os dados relacionados com perceptividade (P1- P5 - variando de lúcido a com ausência de piscamento à ameaça), reatividade inespecífica (R1- R3 - resposta a estímulos verbais), reatividade específica à dor (D1- D4 - resposta a dor) e reatividade autonômica (V1-V2 - presença de sintomas autonômicos) ${ }^{10}$. É ideal para acompa-nhamento de pacientes em recuperação funcional após quadros neurológicos graves.

\section{Padrão respiratório}

A respiração normal depende da integração de dois componentes: o centro respiratório, localizado no tronco encefálico entre a ponte e a junção bulbo-cervical, responsável pela regulação da oxigenação e do balanço ácido-básico; e o componente prosencefálico que regula os aspectos comportamentais, relacionados, por exemplo, a produção da fala. As informações integradas nesses dois sistemas influenciam, através de fibras descendentes, os motoneurônios espinhais relacionados com os músculos da respiração ${ }^{1}$.

Nos pacientes em coma, vários padrões respiratórios foram descritos e podem nos auxiliar no diagnóstico topográfico da lesão. Entre eles podemos incluir: respiração do tipo Cheyne-Stokes, hiperventilação neurogência central, apnêustica, salvas ("cluster") e atáxica ${ }^{1,3}$.

O padrão do tipo Cheyne-Stokes consiste na oscilação lenta entre a hiperventilação e hipoventilação, intercalados por períodos de apnéia ${ }^{1,3,11}$. Após um período de apnéia, ocorre um aumento gradual da amplitude do movimento respiratório e hiperventilação (em crescendo) até atingir um pico, seguido por um período de diminuição da amplitude respiratória e hipoventilação (em decrescendo). Pode ser encontrada em pacientes com lesões hemisféricas bilaterais ou insultos diencefálicos, bem como, em lesões bilaterais entre o prosencéfalo e a região superior da ponte. Os pacientes com insuficiência cardíaca grave, devido ao prolongado tempo de trânsito circulatório, podem apresentar esse padrão respiratório e devem ser diferenciados apropriadamente.

A respiração do tipo neurogênica central é caracterizada por um quadro de hiperventilação rápida e sustentada, em geral com freqüência respiratória entre 40 e 70 por minuto, observada em pacientes com lesões do tegumento pontino central, ventral ao aqueduto ou ao quarto ventrículo ${ }^{1,3}$. Deve-se ser diferenciada da hiperventilação reativa secundária a alterações metabólicas da hipoxemia devido a comprometimento pulmonar.
A respiração apnêustica consiste em uma respiração profunda com pausa de 2 a 3 segundos no final da inspiração. Pode ser observado em lesões na região dorso-medial da metade inferior da ponte ${ }^{1,3}$. Em geral, associada a infarto pontino causado por trombose da artéria basilar.

A respiração do tipo em salvas ("cluster") caracteriza-se por movimentos respiratórios periódicos de amplitude e freqüência irregulares, intercalados por pausas respiratórias ${ }^{1,3}$. Pode ser observada em pacientes com lesões na porção inferior da ponte e superior do bulbo.

A respiração do tipo atáxica é caracterizada pelo padrão irregular da freqüência, ritmo e amplitude respiratória. Respirações profundas e superficiais de diferentes amplitudes e ritmos alternam-se ao longo dos ciclos respiratórios, podendo haver inclusão de pausas respiratórias ${ }^{1,3}$. As lesões geralmente acometem o bulbo. A associação desse padrão respiratório e paralisia do VI nervo craniano podem indicar compressão do tronco cerebral por lesões expansivas da fossa posterior.

\section{Tamanho pupilar e reatividade a luz}

O tamanho e reatividade pupilar são dependentes da ação dos neurônios simpáticos e parassimpáticos que inervam os músculos dilatadores e constrictores da pupila. O sistema simpático estimula a contração dos músculos dilatadores da pupila, determinando midríase e o sistema simpático a contração dos músculos constrictores da pupila, levando a miose. Em repouso, há uma ação tônica contínua tanto do sistema simpático e parassimpático. Se houver comprometimento de uma das vias simpática ou parassimpática, o efeito no tamanho da pupila, miose ou midríase, dependerá da ação do sistema menos acometido ou intacto. Portanto, lesões em neurônios da via simpática levam a miose e da via parassimpática a midríase ${ }^{1}$.

A via simpática pupilo-dilatadora tem origem no hipotálamo e apresenta fibras descendentes que atravessam todo o tronco encefálico até a coluna intermédio-lateral da medula espinhal torácica. Fibras pré-ganglionares simpáticas deixam a medula espinhal e fazem sinapse no gânglio cervical superior. Fibras pós-ganglionares simpáticas, através do nervo nasociliar, inervam os músculos pupilo-dilatadores ${ }^{1}$.

A via parassimpática aferente inicia-se nas células ganglionares retinianas e segue através do nervo e trato óptico até chegarem na região pré-tectal. A via aferente manda informações para os núcleos pré-tectais bilateralmente, que estimulam os neurônios localizados no núcleo de Edinger-Westphal 
correspondentes. A via eferente segue através do nervo óculo-motor a partir do núcleo de EdingerWestphal localizado no mesencéfalo, e vai inervar os músculos pupilo-constrictores. O reflexo pupilar consiste na contração pupilar após estímulo luminoso, através da via parassimpática ${ }^{1}$.

Como as áreas relacionadas ao controle da consciência encontram-se adjacentes a essas vias, as alterações pupilares podem nos auxiliar na diferenciação e localização das possíveis causas de coma $^{3}$.

Lesões hipotalâmicas, principalmente na região posterior e ventrolateral, podem produzir constrição pupilar ipslateral, geralmente associado com ptose e anidrose (Síndrome de Horner). Lesões talâmicas podem levar a pupilas pequenas e reativas, chamadas de pupilas diencefálicas.

Lesões mesencefálicas podem produzir três tipos de alterações pupilares dependendo da localização da lesão: lesões nas regiões tectais dorsais, que interrompem a reação pupilar à luz, levando a pupilas médias ou pouco dilatadas ( $5 \mathrm{a} 6 \mathrm{~mm}$ ), fixas e com preservação do reflexo de acomodação. Pode ocorrer flutuação do tamanho pupilar (hippus) e há manutenção do reflexo cílioespinhal; lesões nucleares mesencefálicas, geralmente comprometem tanto a via simpática quanto parassimpática, levando a pupilas médio-fixas (4 a $5 \mathrm{~mm}$ ), geralmente pouco irregular, geralmente relacionadas a herniação transtentorial; lesões do III nervo craniano bilateral, levam a midríase paralítica, geralmente relacionada a herniação uncal ${ }^{1,3}$.

Lesões no tegumento pontino interrompem as vias descendentes simpáticas levando a miose bilateral com preservação do reflexo pupilar a luz. Pupilas puntiformes devido a hemorragias pontinas podem resultar da lesão das vias simpáticas e irritação das vias parassimpáticas ${ }^{1}$.

Nos pacientes em coma relacionados a alterações tóxico-metabólicas em geral as pupilas são pequenas e reativas à luz ${ }^{1,3}$. As ações de drogas sejam locais ou sistêmicas, podem levar a alterações pupilares devido ao efeito dessas sobre as vias simpáticas ou parassimpáticas. As drogas anticolinérgicas, como atropina ou escopolamina, podem levar a dilatação pupilar. Opiáceos (heroína e morfina) podem levar a miose, semelhante à hemorragia pontina. Barbitúricos podem determinar pupilas fixas. Os dados de história clínica e outros dados do exame neurológico podem nos auxiliar na diferenciação desses casos.

\section{Motricidade ocular}

A motricidade ocular depende da integridade de estruturas localizadas no cérebro, cerebelo e tronco cerebral ${ }^{3}$. Como no paciente em coma não podemos avaliar a motricidade ocular voluntária, devemos avaliar a integridade das vias reflexas localizadas no tronco cerebral. A ausência de alterações na motricidade ocular significa que a região localizada entre os núcleos vestibulares na junção bulbo-pontina até os núcleos óculo-motores mesencefálicos estão intactos ${ }^{3}$.

No doente em coma devemos avaliar três aspectos com relação a motricidade ocular: (1) olhar primário em repouso, para avaliar a presença de desvios; (2) observar a presença de movimentos oculares espontâneos e (3) testar os movimentos oculares reflexos ${ }^{3}$.

A presença de desvios na posição de repouso pode nos trazer indícios de paralisias de nervos cranianos, em geral quando há um desvio desconjugado do olhar. Desvios conjugados do olhar lateral podem ser resultados de lesões desde o córtex até a formação reticular parapontina contralateral. Desvios desconjugados do olhar lateral podem ser reflexo de paralisia do nervo abducente, óculo-motor ou oftalmoplegia internuclear. A presença de desvio dos olhos para baixo pode significar lesões do tronco encefálico, como a compressão do tecto mesencefálico. Lesões talâmicas e subtalâmicas podem levar tanto a desvios conjugado dos olhos para cima quanto para baixo. Outras condições que podem levar a desvio dos olhos para cima são sono, crise epiléptica, síncope, apnéia da respiração de Cheyne-Stokes, hemorragia no vérmis cerebelar, isquemia ou encefalite de tronco. $O$ desvio Skew é um desvio em que um dos olhos está acima do outro (hipertrópico), geralmente correspondendo a lesões do tronco cerebral ou cerebelo ${ }^{3}$.

A observação de movimentos oculares espontâneos pode nos trazer informações importantes quanto à integridade das vias óculo-motoras e a topografia das lesões. A presença de movimentos do tipo "roving" (do tipo "perambulando"), que consiste em movimentos conjugados do olhar lateral, lentos e espontâneos, pode indicar a integridade das vias óculo-motoras e de suas conexões. A presença de nistagmo no doente comatoso pode ser indicativo de um foco irritativo ou epileptiforme supratentorial. Bobbing ocular é caracterizado por movimentos conjugados rápidos para baixo seguido de retorno lento para a posição primária, ocorrendo geralmente em lesões do tronco encefálico, especialmente na ponte ${ }^{3}$.

A avaliação dos movimentos oculares reflexos pode ser feito pela manobra óculo-cefálica ou pelo teste calórico (vestíbulo-ocular). A manobra óculocefálica é feita através da rotação lateral ou vertical da cabeça e a observação da movimentação ocular. Em 
pacientes com preservação desse reflexo, os olhos movem de forma conjugada e em direção oposta ao movimento da cabeça ${ }^{1,3,4}$.

O teste calórico consiste na estimulação das vias vestíbulo-oculares através do uso de água morna ou fria, aplicada ao conduto auditivo. A presença de água morna em contato com a membrana timpânica causa reversão do fluxo da endolinfa, o que causa um desvio do olhar conjugado de fase lenta para o lado oposto ao estimulado e um movimento rápido corretivo ou nistagmo em direção ao lado estimulado. O efeito com a água fria é o inverso $0^{1,3,4}$.

Através dessas manobras podemos observar a integridade das vias óculo-motoras no tronco cerebral e evidenciar a paralisia de nervos cranianos isolados. A ausência de resposta reflexa bilateral pode indicar a presença de lesões extensas no tronco encefálico ${ }^{1,3,4}$.

\section{Resposta motora}

O exame motor do paciente em coma é realizado inicialmente através da observação da postura em repouso, da presença de movimentação espontânea, ou da resposta aos estímulos verbais ou dolorosos. A resposta motora deve ser analizada comparativamente com o lado oposto, e após estímulo simétrico nos quatro membros ${ }^{3}$.

Desvio da cabeça e dos olhos para um lado e hemiparesia contralateral indica lesão supratentorial, enquanto o desvio para o mesmo lado da hemiparesia pode indicar lesão de tronco cerebral. Rotação externa do membro inferior pode ser indicativa de hemiparesia ${ }^{1}$.

Ausência ou assimetria de resposta de um lado pode corresponder a hemiparesia e a ausência ou diminuição de resposta nos quatro membros pode corresponder a tetraparesia.

Postura em decerebração consiste em extensão bilateral dos membros inferiores e adução e rotação interna dos ombros e extensão dos cotovelos e punhos. Geralmente significa lesão bilateral em mesencéfalo e ponte, mas pode ser evidenciado em encefalopatias metabólicas graves ou lesões supratentoriais envolvendo o trato cortiço-espinhal bilateral ${ }^{1,3}$.

Postura em decorticação consiste em flexão dos cotovelos e punhos, adução dos ombros e extensão dos membros inferiores. Apesar de não ser uma postura com boa correlação topográfica, geralmente indica lesões acima do tronco encefálico ${ }^{1,3,12}$.

Essas posturas anormais podem ser observadas espontaneamente ou após estímulos dolorosos, e sua presença pode sugerir uma síndrome de herniação do tronco encefálico ${ }^{1,3}$.
Outros movimentos que podem ser observados são: abalos tônico-clônicos nas crises epilépticas seja de forma focal ou generalizada; mioclonias, observado com freqüência em quadros de encefalopatia pós-anóxia e outros comas metabólicos; respostas reflexas, como a resposta em tríplice flexão dos membros inferiores e resposta plantar em extensão ${ }^{3}$.

\section{DIAGNÓSTICO DIFERENCIAL}

Alguns dados do exame neurológico podem nos ajudar a diferenciar o coma tóxico-metabólico do coma relacionado à doença estrutural:

1. Nível de consciência: pacientes com quadros de encefalopatia difusa tóxico-metabólica habitualmente apresentam alterações mais brandas nível de consciência e com tendência a oscilação entre melhora e piora do quadro. Em geral, pacientes com lesões estruturais tendem a apresentar piora ou estabilização do nível de consciência com a evolução do quadro

2. Respiração: respiração profunda e freqüente é mais encontrada em pacientes com encefalopatia metabólica, embora raramente pode ser causada por lesões pontinas ou por edema agudo de pulmão neurogênico secundária a lesão estrutural aguda ${ }^{3}$;

3. Tamanho pupilar e reatividade pupilar: geralmente as pupilas de pacientes com alterações metabólicas são simétricas, pequenas e reativas a luz. Exceção nos quadros de intoxicação por drogas ou encefalopatia anóxica. A reatividade pupilar é um dos parâmetros de maior significado na diferenciação de causas de coma metabólicos e estruturais, já que esse reflexo, em geral, é resistente a insultos tóxicometabólicos ${ }^{3}$;

4. Motricidade ocular: assimetria ou desvios oculares são indicativos de dano estrutural. A presença de movimentos espontâneos oculares do tipo roving pode sugerir causa tóxico-metabólica. Os movimentos oculares reflexos geralmente estão intactos em pacientes com coma tóxico-metabólico ${ }^{3}$;

5. Tônus muscular: assimetria de tônus pode ser indicativa de lesão estrutural ${ }^{3}$;

6. Posturas: posturas em decerebração ou decorticação podem ser indicativos de lesão estrutural ou indicar síndrome de herniação de tronco cerebral. Mioclônias podem sugerir encefalopatias metabólicas ou pós-anóxica ${ }^{3}$;

7. Fundo de olho: hemorragias vítreas ou subhialóideas podem indicar hemorragia subaracnóidea. Papiledema pode indicar hipertensão intracraniana ou encefalopatia hipertensiva ${ }^{3}$. 
Devemos ficar atentos para as causas estruturais que podem mimetizar alterações difusas tóxico-metabólicas como: hemorragia subaracnóide, trombose venosa cerebral, hematoma subdural crôni- co bilateral e meningite ${ }^{1,3}$. Causas tóxico-metabólicas também podem simular causas estruturais focais, como por exemplo, intoxicações por barbitúricos, hipoglicemia, encefalopatia hepática e hiponatremia ${ }^{1,3}$.

Tabela 1. Causas de alterações do nível de consciência e coma

\begin{tabular}{l|l}
\hline Supratentoriais & TCE \\
& Tumor ou abscesso cerebral \\
AVC & Hematoma Epidural ou Subdural \\
\hline Infratentoriais & $\begin{array}{l}\text { AVC isquêmico ou hemorrágico de tronco ou cerebelo, } \\
\text { tumor, trauma ou hemorragia }\end{array}$ \\
\hline Desordens cerebrais difusas e metabólicas & $\begin{array}{l}\text { Anóxia ou isquemia (síncope, arritmia cardíaca, tromboembolismo } \\
\text { pulmonar, insuficiência respiratória, intoxicação por monóxido de }\end{array}$ \\
& $\begin{array}{l}\text { carbono) } \\
\text { Distúrbio hidroeletrolítico (uremia, insuficiência hepática, cetoacidose } \\
\text { diabética, hipoglicemia, hiponatremia) } \\
\text { Intoxicação exógena (barbitúricos, etanol, metanol, morfina, heroína, } \\
\text { hipotermia) } \\
\text { Epilepsia } \\
\text { Estado pós-ictal }\end{array}$ \\
\hline Distúrbios psiquiátricos & Catatonia \\
& Histeria \\
\hline
\end{tabular}

1. Abertura ocular: $\quad \begin{aligned} & \text { 2espontânea } \\ & \text { 2. Resposta verbal: }\end{aligned}$

Figura 1. Escala de Coma de Glasgow 


\section{Tabela 2. Escala de Coma de Jouvet modificada}

Lúcido, obedece a ordens complexas, até escritas $\quad \mathrm{P}_{1}$

Desorientado (obnubilado) e não obedece a

comandos escritos

Obedece apenas ordem verbal (estado de torpor)

Apresenta apenas piscamento à ameaça (blinking)

Sem resposta

\section{REATIVIDADE INESPECÍFICA}

(reflexo cócleo-palpebral)

Aos estímulos verbais, acorda e orienta

Aos estímulos verbais, só acorda

Sem resposta

$R_{2}$

$\mathrm{R}_{3}$

\section{REATIVIDADE ESPECÍFICA (à dor)}

Acorda, retira, mímica, vocaliza

Acorda e retira, sem mímica e vocalização

Retirada inespecífica

Padrão decorticação

Padrão descerebração

Sem resposta

\section{REATIVIDADE AUTONÔMICA (à dor)}

Taquicardia, taquipnéia, midríase

Sem resposta

P4 - R2-D4 a D6-V1 = Estado Vegetativo Persistente

P5 - R3 - D6 - V1 = Coma 3 pontos não apneico

P5 - R3 - D6 - V2 = Coma 3 pontos apneico (morte

encefálica ?)

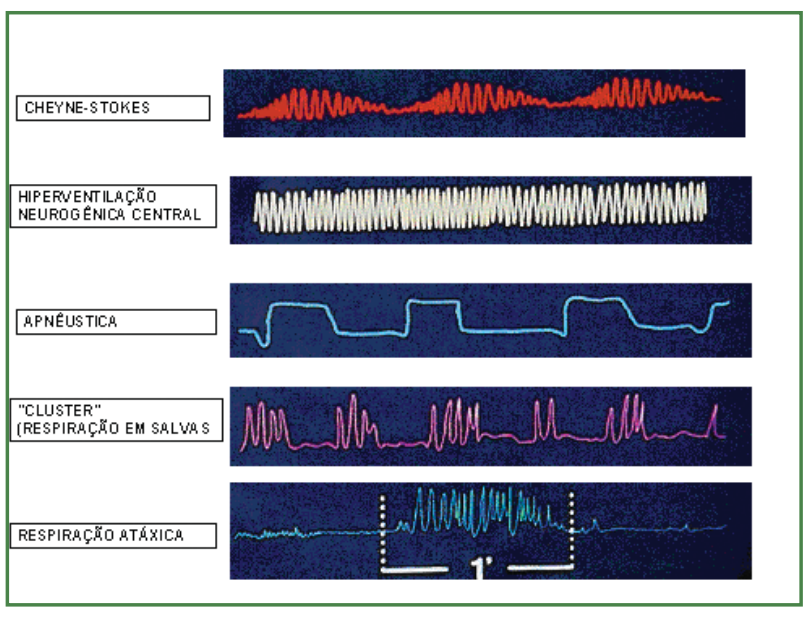

Figura 2. Padrões respiratórios no coma

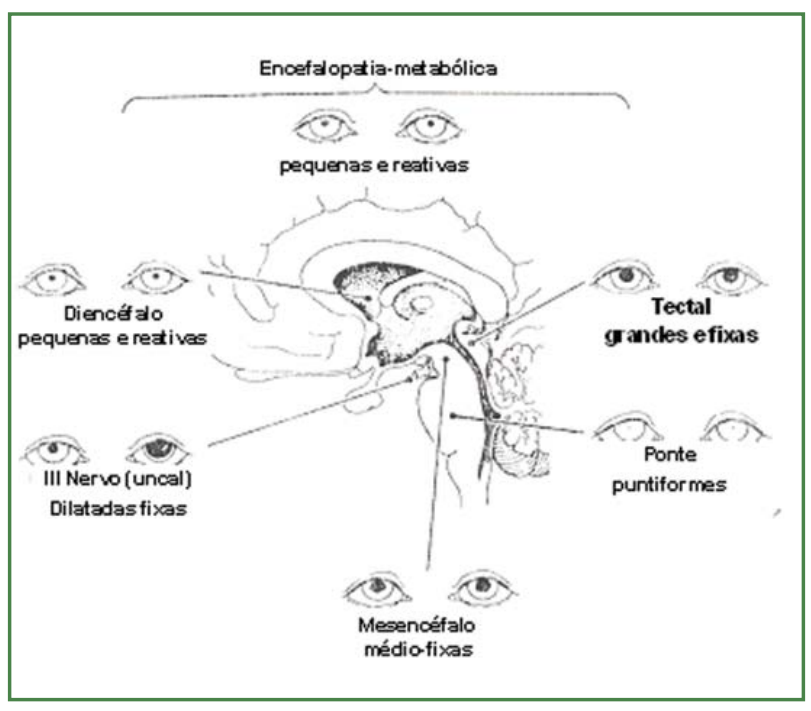

Figura 3. Aspectos patológicos das pupilas no coma

Andrade AF de, Carvalho RC, Amorim RLO de, Paiva, WS, Figueiredo EG, Teixeira MJ. Coma and other consciousness disorders. Rev Med (São Paulo). 2007 jul.-set.;86(3):123-31.

ABSTRACT: The alterations of the level of conscience can vary among two ends, from a mental confusion to a state of deep coma. In clinical practice it is extremely common a patient with alteration of the level of conscience without diagnosis. In general hospitals, studies indicate that the alcoholism, the brain trauma and the cardiovascular diseases are the main causes of coma $(82 \%)$. Its necessary know how to recognize the main causes of unconsciousness of and their differential diagnosis, aimed to identify the severe ill patient and to treat the possible emergency states that take to a secondary neurological damage. In this revision, we emphasized the key points in the neurological exam to determine the different alterations of the level of consciousness.

KEY WORDS: Coma/diagnosis. Coma/physiopathology. Brain injuries/etiology. Consciousness/classification. Diagnosis, differential. 


\section{REFERÊNCIAS}

1. Plum F, Posner JB. The pathologic physiology of signs and symptoms of coma. In: Plum F, Posner JB. Diagnosis of stupor and coma. 3rd ed. Philadelphia: Davis CO; 1984. p.1-86.

2. Plum F, Posner JB. Brain death. In: Plum F, Posner JB. Diagnosis of stupor and coma. 3rd ed. Philadelphia: Davis; 1984. p.313-28.

3. Berger JR. Clinical approach to stupor and coma. In: Bradley WG, Daroff RB, Fenichel GM, Jankovic J. Neurology in clinical practice. Principles of diagnosis and management. 4th ed. Salt Lake: Elsevier; 2004. v.1, p.43-64.

4. DeJong RN. The examination in coma, in other states of disordered consciousness, and in brain death. In: De Jong RN. The neurologic examination. 5th ed. Philadelphia: Lippincott; 1992. p.483-88 .

5. Kaufman HH, Brick J, Frick M. Brain death. In: Youmans JR. Neurological surgery: a comprehensive reference guide to the diagnosis and management of neurosurgical problems. Philadelphia: WB Saunders; 1996. p 439-51.
6. Teasdale G, Jennett B. Assessment of coma and impaired consciousness. A practical scale. Lancet 1974;2:81-4.

7. Jennett $B$. The history of the Glasgow coma scale: an interview with professor Bryan Jennett. Interview by Carole Rush. Int J Trauma Nurs. 1997;3(4):114-8.

8. Teasdale GM, Murray L. Revisiting the Glasgow coma scale and coma score. Intensive Care Med. 2000;26(2):153-4.

9. Sternbach GL. The Glasgow coma scale. J Emerg Med. 2000;19(1):67-71.

10. Jouvet $M$. Coma and other disorders of consciousness. In: VinkenPJ, Bruyn GN. Handbook of clinical neurology. Amsterdam: North Helland Publishing Company; 1969. p.62-79.

11. Cherniack NS, Longobardo G, Evangelista CJ. Causes of Cheyne-stokes respiration. Neurocrit Care. 2005;3(3):271-9.

12. Sabiar TD. Coma and the acute confusional state in the emergency room. Med Clin North Am. 1981;65:15-7. 